\title{
ADVANCED INFORMATION TECHNOLOGY AND MANAGEMENT IN CONSTRUCTION
}

\author{
Thomas Bock, Friedrich Gebhart \\ Division for Automation in Construction \\ Institute for Mechanical Engineering in Construction-Management \\ Werner Steck \\ Institute for Machine Tools and Production Science \\ University of Karlsruhe, Am Fasanengarten, 7500 Karlsruhe, Germany
}

\begin{abstract}
The industrialization of the building industry has opened a new dimension by the introduction of advanced information technologies offering new possibilities for the building process. Due to the increasing market requirements and nowadays available technological potentials, traditional building production will be replaced by one-of-a-kind computer integrated construction systems. The use of modern information technology in planning, pre-production and assembly on-site can have several consequences to building. By means of simultaneous planning, production and assembly, the capacity as well as quality of construction and building can be improved. The problems of last minute changes during design and build process can be avoided by an advanced information technology and management system.
\end{abstract}

\section{INTRODUCTION}

The progress of productivity in the construction industry is relatively slow compared to other fields of the manufacturing industry. Reasons are mainly single piece production, complex tasks, and constantly changing environments with every new building task, e.g., the very individual designs of apartment houses with angled and curved walls or bay windows.

\section{Traditional house building process}

The conventional construction is still characterized by high manual work intensity, difficult working conditions and low productivity. In order to describe the present situation and to demonstrate future developments, masonry was chosen as an example. The described contents can easily be transfered to other activities on construction site. To define the tasks, which must be fulfilled by an automated system, one first has to identify the conventional method of masonry construction. The traditional building materials are bricks and mortar. The bricks must meet the requirements of load bearing capacity, heat and noise insulation, and protection against other environmental influences. The mortar is basically used as a levelling layer because of the inaccuracy of the bricks. The traditional mason tools are bubble level, plumb, batter cord and a hammer, which are used to get a vertically and horizontally correct wall at the right place with the right length. The building method is to level out each brick in a mortar bed with these tools and, if necessary, to cut the brick with the hammer into the right shape. These materials, tools, and methods have remained unchanged for centuries.

\section{From Mechanized to Robotized House Building}

The conventional masonry process requires highly skilled workmen in order to achieve sufficient and consistent walling quality. This labour intensive construction process results in relatively high costs. To achieve higher rationalization and humanization, different approaches are currently being
tested. 
One of the approaches, which is currently used by the sandlime brick and cellular concrete industry in Europe, is the enlargement of the building block sizes. The larger dimensions of these blocks, however, coincide with their increased physical weight of up to $300 \mathrm{~kg}$. Due to larger dimensions and heavier weights these building blocks are not ergonomically desirable and, therefore, various mechanical aids such as hydraulic balancers or minicranes with counterweights are used.

A second approach is the pre-fabrication of customized block in the factory. This requires positioning plans for the on-site assembly, which are already generated with the help of CAD/CAMsystems. The information was also used to program the in-factory cutting equipment to customize the blocks. The pre-cutting and the assembly plans enhance the working speed on-site, significantly, and also help to reduce the complexity of the masonry task.

Another tendency, which is steadily gaining momentum, is the use of more exact, plane-parallel blocks, which are layed in thin-bed mortar/glue or, as shuttering blocks, are assembled dry and filled with concrete after the assembly of the whole wall. These methods result in better physical properties because of the reduction of walling operations because of no mortar joint, enabling higher working speed and easier use.

The differing accuracies of below grade and of walling work are usually compensated for by a first layer of smaller blocks in a more or less thick mortar bed. By this method, the difficult alignment, which is required for adjusting the position of each block, can be reduced. Even though an increased working speed can be achieved while employing less qualified workers on the construction site, the present and future share of cost due to labour will be constantly increasing.

Further rationalization can only be achieved by reduction of labour and construction time. Since the above mentioned mechanised methods for masonry have reached their systems limits, they cannot contribute to further effectiveness enhancement. Therefore, a certain innovative leap is required by a systems approach that synchronizes existing construction material with new information technology (IT) enhanced and semi-autonomous robotic technologies which are designed for construction site application.

The system approach of the University of Karlsruhe deals mainly with the construction oriented modification of existing technologies and materials, development of construction oriented handling systems. The aim is to close the gaps between these subsystems through intelligent interfaces and ITtools, in order to provide the necessary flexibility for one of a kind building production, robust design, and user friendly programming.

The advantage of on-site mechanization and future on-site automation compared to a stationary prefabrication is higher flexibility relative to the materials, damaged elements and (important for the building industry) short term market changes.

\section{Present Information Technology and Management}

The implementation of CAD-programs in architectural offices is very heterogenous. Many different systems are currently in use in the bigger offices, while the smaller ones are still working by hand to produce the design plans.

The hand/CAD made design plans are used by the construction companies to order the necessary materials from the building material manufacturers and to cut, trim and assemble the blocks on-site (fig 1). 


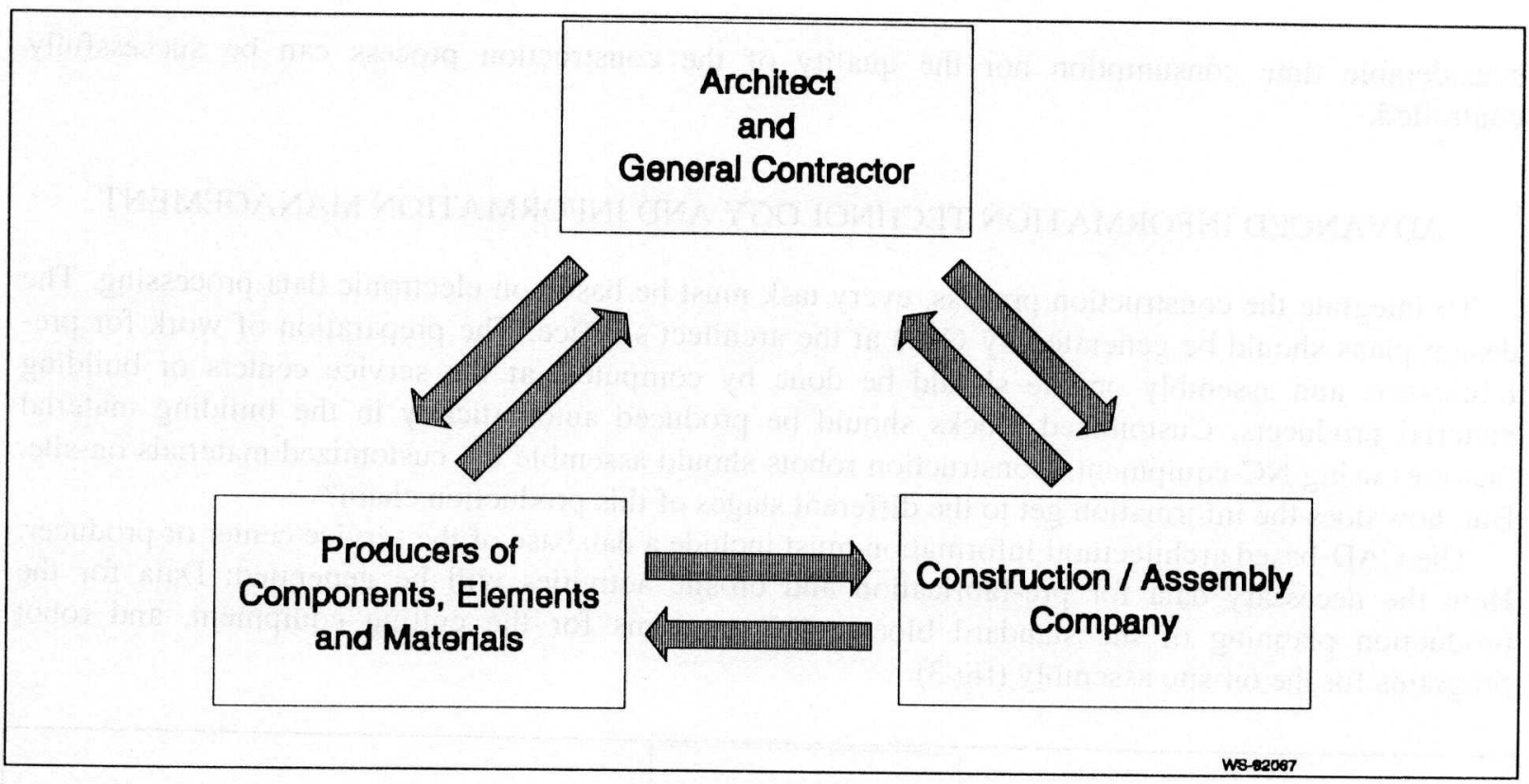

Fig. 1: Structure of present information management

The present structure of information management combined with the today usual conventions force a sequential and time consuming construction process (fig. 2).

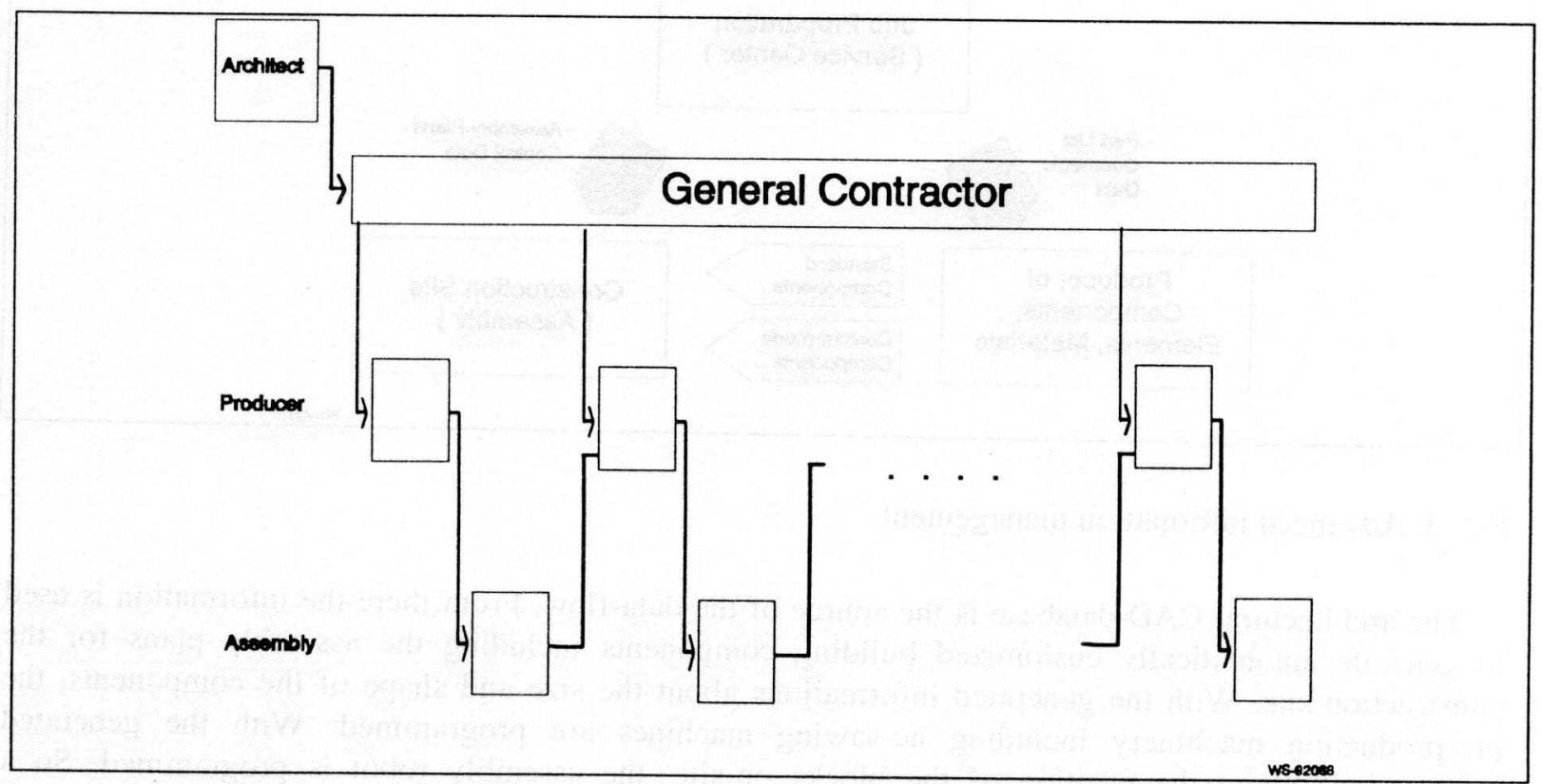

Fig. 2: Sequential construction process

The traditional conventions of sequential construction operations originate from the varying accuracies of the diversified construction processes. In order to compensate the less accurate foundation, concrete or mason works, the subsequent operations usually measure the accuracies of these previous works and then start the execution of their proper construction operation. Neither the 
considerable time consumption nor the quality of the construction process can be successfully
controlled.

\section{ADVANCED INFORMATION TECHNOLOGY AND INFORMATION MANAGEMENT}

To integrate the construction process, every task must be based on electronic data processing. The design plans should be generated by CAD at the architect's office. The preparation of work for prefabrication and assembly on-site should be done by computers at the service centers or building material producers. Customized blocks should be produced automatically in the building material factories using NC-equipment. Construction robots should assemble the customized materials on-site. But, how does the information get to the different stages of this production chain?

The CAD-based architectural information must include a database of the service center or producer. Here the necessary data for pre-fabrication and on-site activities will be generated: Data for the production planning of the standard blocks, NC-programs for the cutting equipment, and robot programs for the on-site assembly (fig 3).

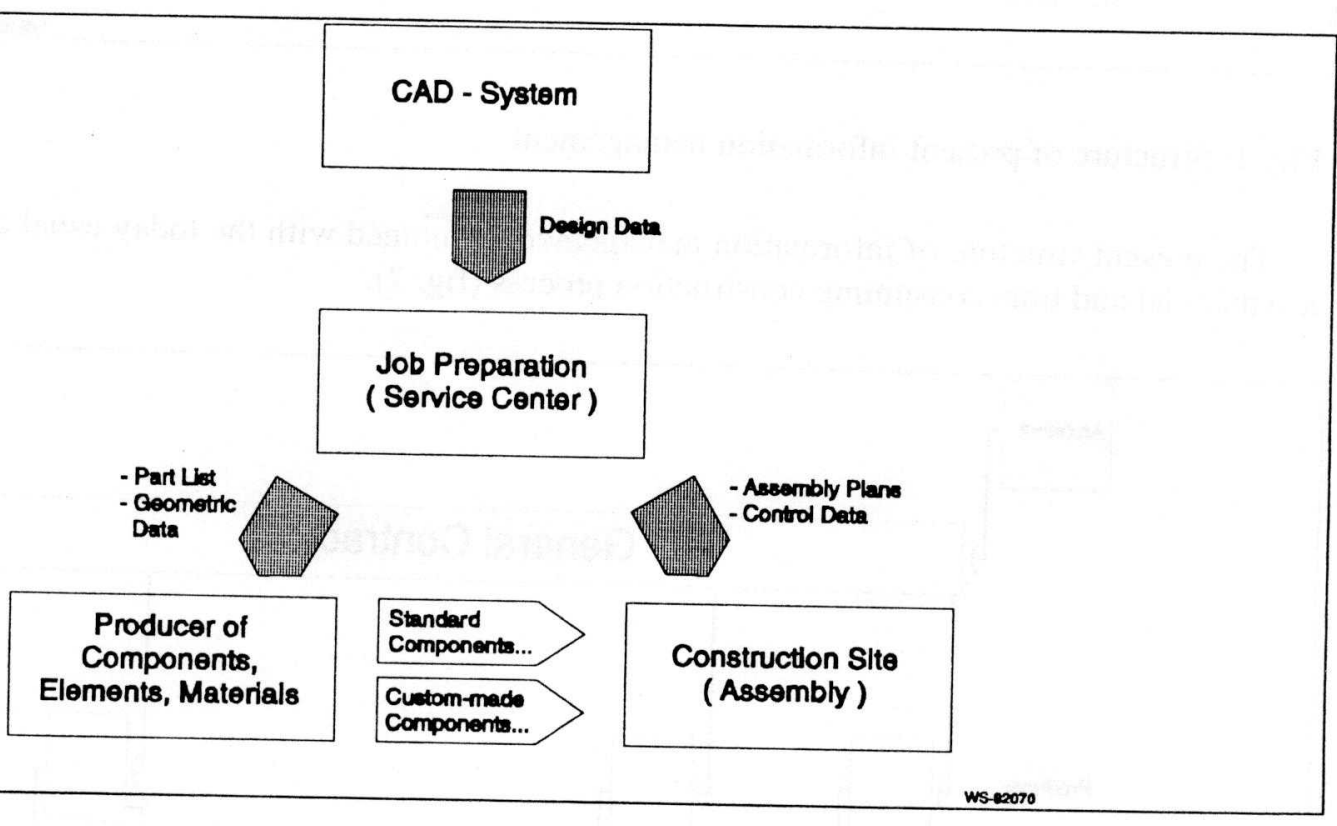

Fig. 3: Advanced information management

The architectural CAD-database is the source of the data-flow. From there the information is used to generate automatically customized building components including the assembly plans for the construction site. With the generated informations about the size and shape of the components, the pre-production machinery including nc-sawing machines are programmed. With the generated informations about the position of the blocks on-site, the assembly robot is programmed. So a continuous data flow from the design through the assembly is realized. Combined with a coordination and harmonization of the accuracies, a parallelization of the construction process becomes possible
(fig 4). 


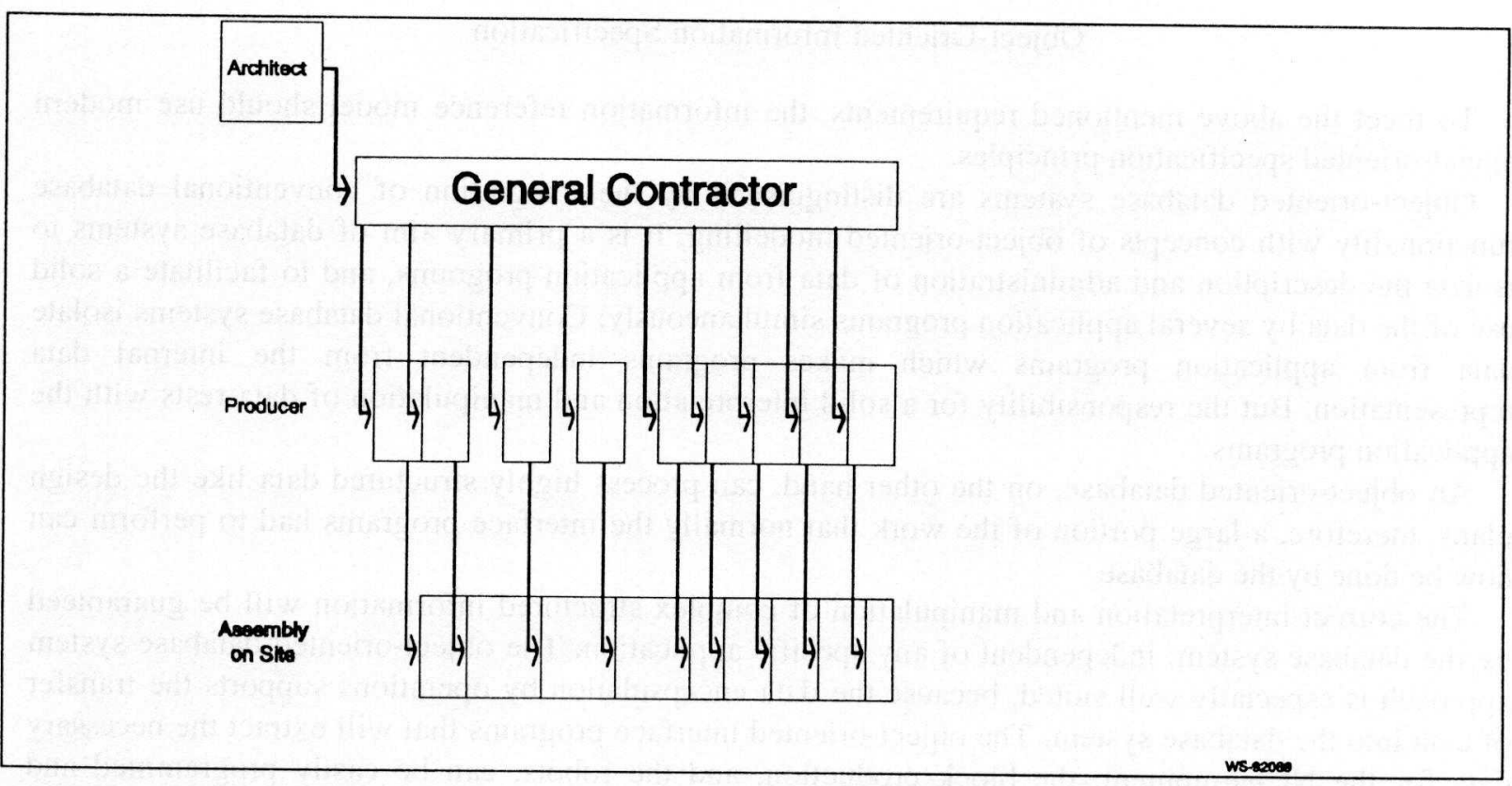

Fig. 4: Parallel construction process

Design and Build Reference Model

The base for an information management is not only a design and build reference model with the aim to describe neutral interfaces between the CIC moduls, but also to provide a link between specific enterprise data and more generic data processed by CIC moduls. Therefore, the following requirements have to be met to ensure information continuity, consistency and rearrangement:

- extentability of the construction model for future CIC moduls

- independency of implementation solution

- maintainability of construction model

- support of tayloring the general CIC moduls towards specific enterprise needs

- sufficient consistency with standardization developments.

This design and build reference model provides an integration frame work for

- product data (geometry, physical qualities)

- production data (in-factory production process, on-site assembly process)

in order to support design and build as well as construction feed back information integration for the planning and the construction of the building.

For the product data, the following major information categories are addressed:

- building element

- building element representation

- building element tolerances

- reference building element

- material and finish properties

These above mentioned features play an important role in the coordination of these information categories. 


\section{Object-Oriented Information Specification}

To meet the above mentioned requirements, the information reference model should use modern object-oriented specification principles.

Object-oriented database systems are distinguished by the integration of conventional database functionality with concepts of object-oriented modelling. It is a primary aim of database systems to isolate the description and administration of data from application programs, and to facilitate a solid use of the data by several application programs simultaneously. Conventional database systems isolate data from application programs which makes programs independent from the internal data representation. But the responsibility for a solid interpretation and manipulation of data rests with the application programs.

An object-oriented database, on the other hand, can process highly structured data like the design plans, therefore, a large portion of the work that normally the interface programs had to perform can now be done by the database.

The correct interpretation and manipulation of complex structured information will be guaranteed by the database system, independent of any specific application. The object-oriented database system approach is especially well suited, because the data encapsulation by operations supports the transfer of data into the database system. The object-oriented interface programs that will extract the necessary data for the NC-equipment, the block production, and the robots, can be easily programmed and changed, because they are independent from the internal data representation in the object oriented database. The often encountered problem of changing data formats can be easily handled by the object-oriented database. Thus an object-oriented database helps to avoid many problems occuring usually with relational databases.

\section{Robot Program Generation}

In one-of-a-kind production like the construction business, the programming is a critical key factor of the rentability of automated equipment. Therefore, an easy to use and as highly as possible automated programming interface must be provided.

Because of the single piece production and constantly changing requirements, normally used programming of robot work cycles cannot be applied. To minimize the individual robot programming efforts, an automatic generation of control data from the existing geometrical data is necessary. This requires completely new concepts of off-line programming, which must integrate as much preplanning as possible and as much on-site flexibility as necessary.

The whole implicit programming task can be subdivided into several subtasks. Three major positioning tasks can be identified after the partitioning into single building components and the custom made pre-fabrication in the factories: First the position of the components on the transport pallets, second the position of the pallets on the site, third the position of each component on the site.

Different restrictions and requirements of the overall system must be considered by the solution of each task. Therefore, we chose an integral approach where an object-oriented database provides the common platform to solve the different problems identified above by different application programs.

For the first application of component palletizing, the following requirements have to be considered:

- Every component must be reachable taking into consideration the robot kinematics, the grippers, and the assembly order of the different components.

- A load capacity optimization of each pallet is necessary.

- The position accuracy of the components on the palets must be considered under the aspect of gripping as well as under the aspect of transport security.

The second application (positioning of the pallets on-site) has to take into account the following requirements and restrictions: 
- Dynamic obstacle avoidance through the generated robot paths and the previously assembled walls must be implemented.

- The robot path should also be minimized by proper positioning of the pallets on the site accounting for the assembly order.

For the third application, emphasis is placed on the following key factors to meet the requirements of the construction site:

- Sensor integration is a key approach for the uncertain environment on construction sites.

- In order to allow hybrid control strategies, robot-control modularity must be provided.

- Fault tolerance of the system is necessary, especially in case of inaccurately positioned or damaged blocks.

The above mentioned advantages of an object oriented approach can also be used in the applications programming. The properties of object orientation like data encapsulation, polymorphism, inheritance and genericity are also useful to solve the optimization, positioning and sensor integration problems occuring in the implicit robot programming.

\section{Towards a Dynamic Information Management System}

Large scale pre-fabrication facilities have reached a high level of automation. Even though the productivity of a factory itself might be very high, its total production system, however, is vulnerable to economic fluctuations due to the tied in capital. Figure 5 shows the idealized fault free system:

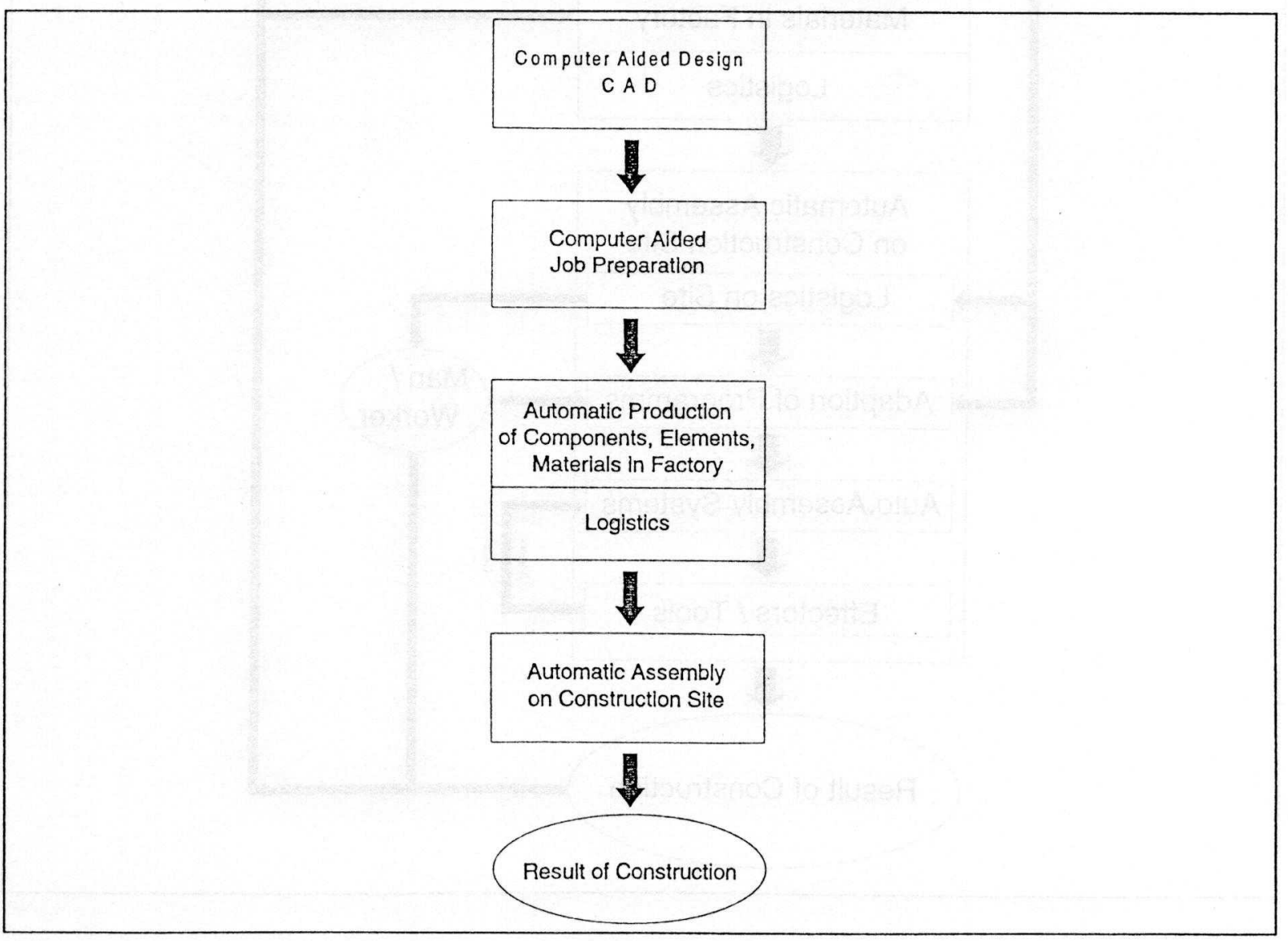

Fig 5: Fault Free "Idealized" System 
In reality, however, some disturbances may occur:

- production faults

- faults in palletizing

- material faults

- damages during transport

- positioning faults at the building-site (pallets, components, robots)

Disturbances of this kind can only be avoided by means of appropriate process control with feedback (fig 6).

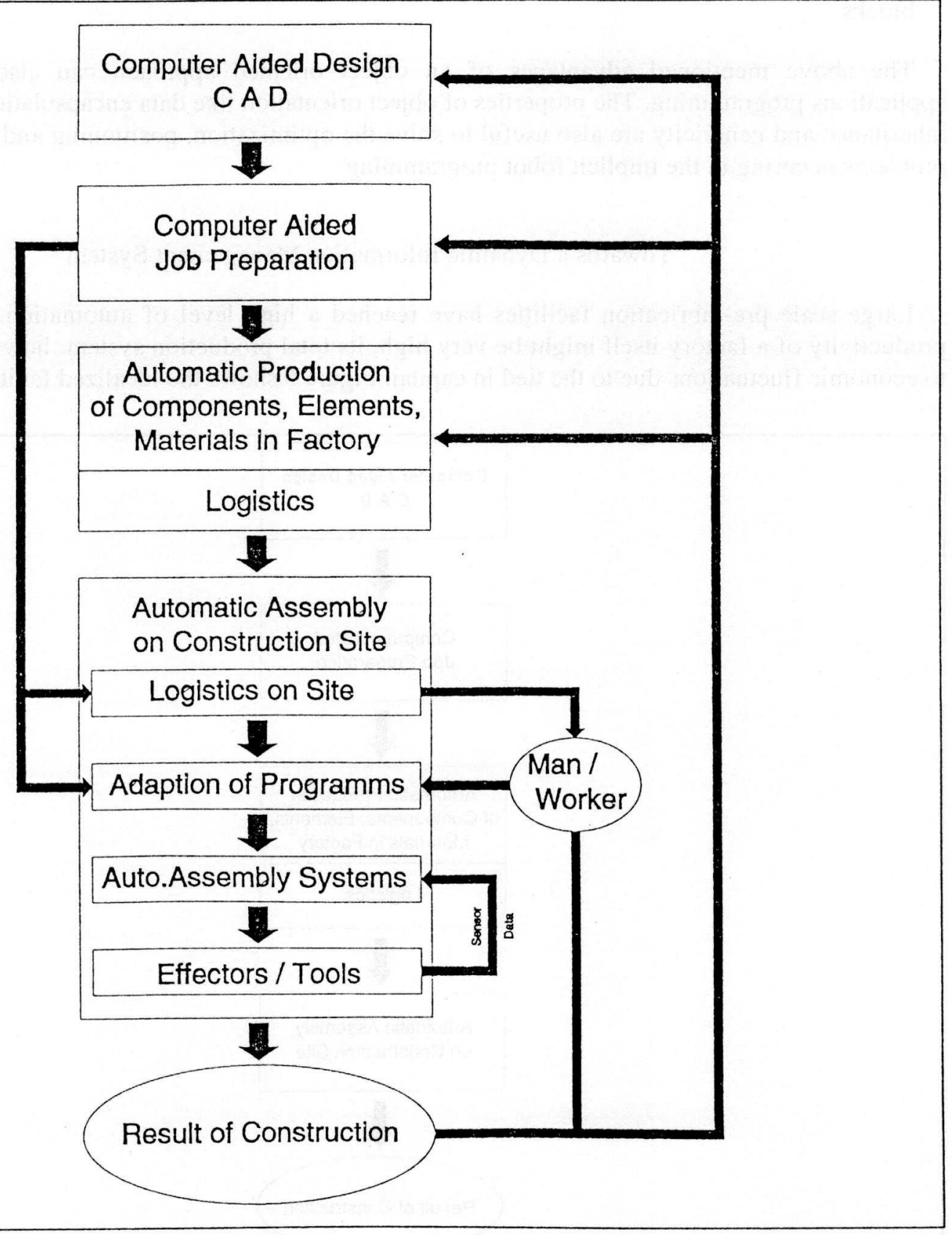

Fig. 6: Exception Control 
Some requirements of the dynamic construction process are as follows:

- feedback of sensor data to the robot

- manual interference in the automatic control mode by remote control

- feedback to previous operations

\section{Feedback of sensor data to the robot}

In case of small positioning faults of the pallets, robots, or the working surface, sensors can perceive these faults. The robot has to be corrected online accordingly.

Disturbances of this kind normally lead only to a small deviation of the gripping areas and effective surfaces.

Disturbances caused by material faults must only be recognized. The same applies if the positioning faults are too considerable. Material faults exclude online corrections because, in this case, an exchange of components would be necessary.

\section{Manual interference in the automatic control mode by remote control}

If the positioning accuracy is too low, or redone components are incorporated, manually remotecontrolled corrections of the process are necessary.

- Transition from automatic to manually remote controlled operation

- Manual remote controlled process

- Transition to automatic operation

The problem consists in the transition to the automation, because this is not a periodically repetitive process. The progress of the working process can not be recognized. Therefore, a hybrid control concept is required which is called on-site programming (OSP).

\section{Feedback to previous operations}

In case of material faults new fabrication is necessary or in case of an existing building material warehouse an additional material supply might be adequate. Furthermore, the technological flexibility has to be supplemented by a flexible information management system on the level of enterprise strategy in order to ensure the appropriate marketability.

Due to market fluctuations and increasing financing costs of construction projects, the time factor becomes more important:

- Therefore a highly flexible pre-fabrication of building components is required. For the information consistency and continuity of the data flow, a central data base has to be installed.

- In order to avoid the execution of faulty design, the design quality has to be controlled at the stage of the job preparation by means of simulation of the constructability.

- If further problems occur during on-site activities, a quick response is possible, though above mentioned hybrid control.

- For future planning and construction, the gained experience can be stored in knowledge based data systems.

\section{CONCLUSION}

The efficiency of traditional construction systems can hardly be further improved. Therefore, we work on an integrated design, construction and building system which should enable construction time reduction, constant design and construction quality and efficient building operation maintenance and recycling. The notion is an advanced information technology and management system based on 
object-oriented database and hybrid control systems for the operator assisted robotic construction and building system. Some modules of the above mentioned requirements are now carried out.

\section{REFERENCES}

1. Meyer, B.: Object-oriented Software Construction, Prentice Hall International (UK) Ltd., 1990

2. Bock, T., Blaser C., Gebhart, F.: Automatisierungsgerechtes Planen und Konstruieren für computerintegriertes Bauen. in: Bautechnik 69 (1992), No. 3, Ernst \& Sohn Berlin.

3. Bock, T., Gebhart, F., Steck, W.: Automatisierung von Hochbauprozessen. in: Baumaschinentechnik 1, April 1992, Bauverlag Wiesbaden. 